\title{
A Reporter of Local Dendritic Translocation Shows Plaque- Related Loss of Neural System Function in APP-Transgenic Mice
}

\author{
Melanie Meyer-Luehmann, ${ }^{1,2,3}$ Matthew Mielke, ${ }^{1}$ Tara L. Spires-Jones, ${ }^{1}$ Will Stoothoff, ${ }^{1}$ Phill Jones, ${ }^{1}$ Brian J. Bacskai, ${ }^{1}$ \\ and Bradley T. Hyman ${ }^{1}$ \\ ${ }^{1}$ MassGeneral Institute for Neurodegenerative Disease, Massachusetts General Hospital, Harvard Medical School, Department of Neurology, Alzheimer's \\ Disease Research Laboratory, Charlestown, Massachusetts 02129, and ${ }^{2}$ Deutsches Zentrum für Neurodegenerative Erkrankungen and ${ }^{3}$ Adolf-Butenandt- \\ Institute for Biochemistry, Ludwig Maximilians-University, Munich 80336, Germany
}

\begin{abstract}
Although neuronal communication is thought to be summated within local dendritic segments, no technique is currently available to monitor activity in vivo at this level of resolution. To overcome this challenge, we developed an optical reporter of neuronal activity using the coding sequence of Venus, flanked by short stretches of the $5^{\prime}$ - and $3^{\prime}$-untranslated regions from calcium/calmodulin-dependent kinase II $\alpha(\mathrm{CAMKII} \alpha)$. This reporter takes advantage of the fact that CAMKII $\alpha$ mRNA is transported to the dendrite and locally translated in an activity-dependent manner. Using adeno-associated virus, we used this reporter to study neuronal activity in adult mice. Exposure of the mice to an enriched environment led to enhancement of Venus expression in dendritic segments of somatosensory cortex, demonstrating in vivo that dendritic mRNA translocation and local translation occur in response to physiologically relevant stimuli. We then used this system to examine the impact of Alzheimer-related local amyloid- $\beta$ deposits on neural system function to test the hypothesis that plaques are toxic. In APPswe/PS1dE9 (APP/PS1) mice, neurons close to plaques, and dendritic segments close to plaques, both showed diminished fluorescent intensity and therefore neuronal activity. In contrast to wild-type mice, fluorescent intensity in neurons near plaques in transgenic mice did not increase after environmental enrichment. These data indicate that neuronal activity in dendritic segments and neurons in the vicinity of a plaque is decreased compared with wild-type mice, supporting the idea that plaques are a focal lesion leading to impaired neural system function.
\end{abstract}

\section{Introduction}

In vivo functional studies of neurons to date have either relied on population characteristics [e.g., long-term potentiation (LTP) phenomenon] or reflect signal integration at the level of the cell body (e.g., sharp electrode studies; calcium imaging). However, much signal integration occurs at the level of the dendritic segment, yet this cell compartment is difficult to interrogate using physical methods. We report herein an optical method designed to detect activity levels in individual dendritic segments as a first step toward higher resolution mapping of structure-function relationships in intact neural systems and as a means of exploring the functional consequences of local pathological changes. The probe design is based on the observation that some messenger RNAs are translocated to the dendrite and locally translated in an activity-dependent manner. One of the best characterized of these is calcium/calmodulin-dependent kinase II (CAMKII $\alpha$ ),

Received April 24, 2009; revised June 26, 2009; accepted July 7, 2009

This work was supported by National Institutes of Health Grants AG08487 (to B.T.H.) and EB000768 (to B.J.B.). We thank Kathryn Bercury, Hisatomo Kowa, Mirjam Koker, and Anne Thomas for technical help and Erin Schuman for the gift of the CAMKII $\alpha$ GFP construct.

Correspondence should be addressed to Dr. Bradley T. Hyman, Massachusetts General Hospital, Department of Neurology, Alzheimer's Disease Research Laboratory, 114 16th Street, Room 2009, Charlestown, MA 02129. E-mail: bhyman@partners.org.

D01:10.1523/JNEUROSCI.1948-09.2009

Copyright $\odot 2009$ Society for Neuroscience $\quad$ 0270-6474/09/2912636-05\$15.00/0 whose protein is highly enriched at synapses, is regulated by neural activity, and is important in synaptic plasticity, learning and memory (Mayford et al., 1996). The $3^{\prime}$ - and 5' -untranslated regions (UTRs) of CAMKII $\alpha$ encode sequences that direct translocation to the dendrite and prevent translation unless the neuron is active (Mori et al., 2000). Compelling evidence from cell culture experiments support the hypothesis that new protein synthesis can appear in dendrites after a given stimulus (Aakalu et al., 2001; Sutton et al., 2004; Smith et al., 2005), but this has not been examined in the intact brain.

To establish methodology whereby the function of an individual dendrite can be evaluated, we used a neuronal activity reporter in which the coding sequence of Venus is flanked by $5^{\prime}$ - and truncated $3^{\prime}$-UTRs of CAMKII $\alpha$. A CAMKII $\alpha$ UTR Venus adeno-associated virus (AAV) was used to examine in vivo whether its synthesis in neurons and especially dendrites change in response to environmental enrichment. We found increased Venus expression after environmental stimulation, supporting the idea that the probe reflects in vivo neural system activation.

In Alzheimer's disease (AD) as well as in transgenic animal models of $\mathrm{AD}$ pathology, amyloid $\beta(\mathrm{A} \beta)$-containing plaques are fibrillar deposits scattered throughout the neuropil in the cortex. Morphological studies have suggested that amyloid plaques disrupt neural systems by leading to abnormal axonal sprouting (Cotman et al., 1990; Phinney et al., 1999) and by distortion of the 

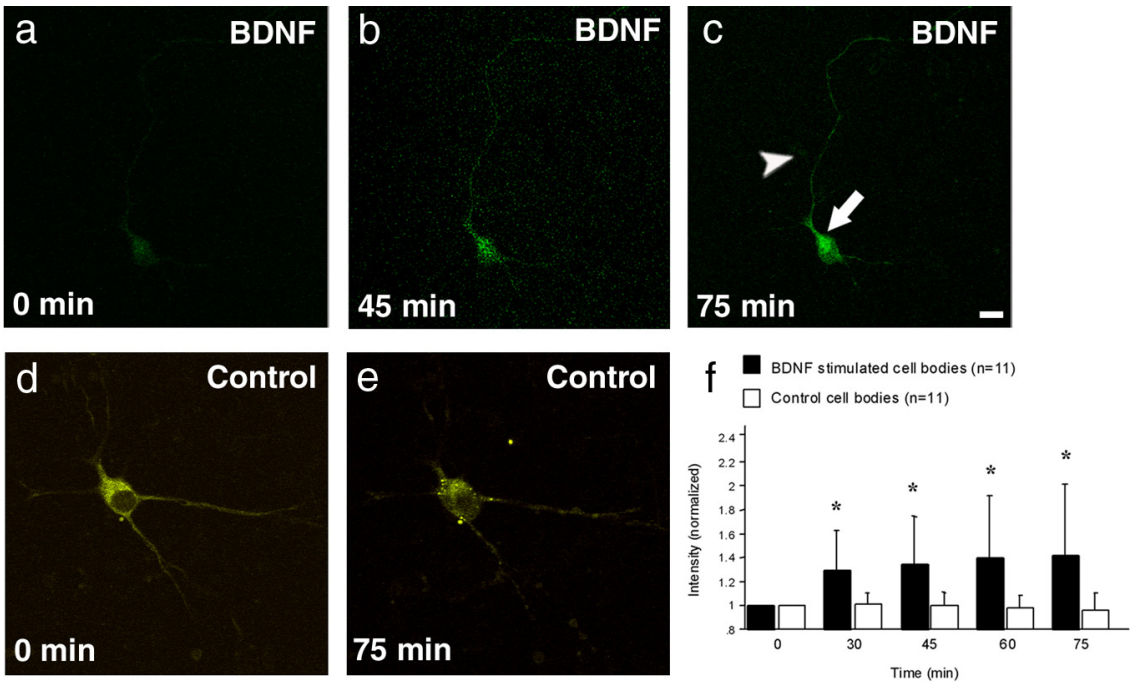

$75 \mathrm{~min}$
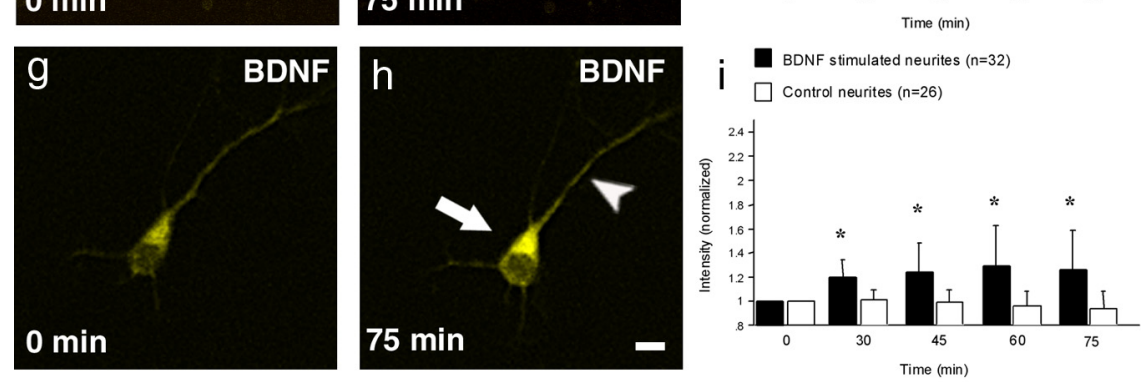

j
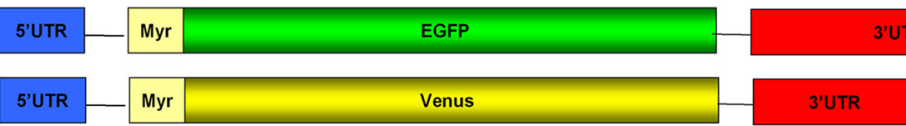

Figure 1. BDNF stimulates protein synthesis of the GFP and Venus reporter in primary neurons. $\boldsymbol{a}-\boldsymbol{c}$, A neuron transfected with $3^{\prime}$-UTR CAMKII $\alpha$ EGFP 5' -UTR reporter and treated for 75 min with BDNF. Before BDNF application, the GFP signal is very modest (a). After 45 and 75 min, the fluorescent signal increases dramatically in the cell body (arrow) as well as in the neurites (arrowhead) $(\boldsymbol{b}, \boldsymbol{c}) . \boldsymbol{d}, \boldsymbol{g}$, An untreated and BDNF-treated cultured neuron expressing the Venus reporter. The BDNF-treated cell shows enhanced fluorescence after $75 \mathrm{~min}$ in the cell body (arrow) and neurites (arrowhead) (h) in contrast to the untreated control cell (e). $\boldsymbol{f}, \boldsymbol{i}$, Summary data for all untreated and BDNF-treated cell bodies and neurites. The intensity remains unchanged in the control neurons and only changes in the stimulated neurons. Note that the intensity is significantly higher after only 30 min of BDNF stimulation in both the cell body $(\boldsymbol{f})$ and neurites $(\boldsymbol{i})$ and increases over time $(* p<0.0001)$. $\boldsymbol{j}$, A schematic representation of the constructs used for this experiment. Scale bars: $\boldsymbol{a}-\boldsymbol{c}, 15 \mu \mathrm{m} ; \boldsymbol{d}, \boldsymbol{e}, \boldsymbol{g}, \boldsymbol{h}, 15 \mu \mathrm{m}$.

dendritic tree (Knowles et al., 1999; Le et al., 2001), yet the functional significance of these changes is unknown. In contrast, recent studies (Shankar et al., 2008) have challenged the idea that plaques are pathophysiological and have even led to the suggestion that they are protective as a depot of otherwise more toxic soluble species of $\mathrm{A} \beta$. We used the CAMKII $\alpha$ Venus reporter to test the hypothesis that plaques focally disrupt neural system function in the cortex. In the setting of microscopic amyloid plaque lesions in APP/ PS1-transgenic mice, the reporter revealed diminished baseline activity and diminished response to environmental enrichment in dendritic segments closest to the amyloid deposits. Our results indicate that neuronal activity is decreased in neurons of these transgenic mice and that their functional neuronal response to environmental stimulation is diminished in the vicinity of plaques.

\section{Materials and Methods}

Cell culture. Primary neuronal cell cultures were prepared from mixed cortical-hippocampal neurons generated from CD1 mice at embryonic day 15-16. Cells were plated on poly-D-lysine-coated coverslipped $35 \mathrm{~mm}$ dishes (MatTek Cultureware) in neurobasal media (Invitrogen) containing 10\% FBS. The neurons were maintained and allowed to mature in neurobasal media containing 2\% B27 supplement (Invitrogen) for 6-12 d before use.
One week after plating the neurons, they were transfected either with the pcDNA3.1-CAMKII $\alpha-5^{\prime}$ UTR-myr.EGFP-3'-UTR, pcDNA3.1-CAMKII $\alpha$ 5'-UTR-myr.Venus-3'-UTR construct or with pcDNA3.1-CAMKII $\alpha$-5'-UTR-myr.Venus construct using Lipofectamine 2000 (Invitrogen) according to the manufacturer's protocol. Forty-eight hours post-transfection, dishes were exposed either to brain-derived neurotrophic factor (BDNF) $(50 \mathrm{ng} / \mathrm{ml})$ or left in their original media, and two to four neurons were imaged per experiment with a Zeiss LSM510 Meta confocal microscope every 15 min up to $75 \mathrm{~min}$. Cells were observed at $63 \times$ for quantification of pixel intensity. In all cell culture experiments, identical acquisition parameters and settings were used for both control and BDNF-treated neurons on a given experimental day. During the experiments, cultured neurons were maintained at $37^{\circ} \mathrm{C}$ and $5 \% \mathrm{CO}_{2}$ level over the entire $75 \mathrm{~min}$ duration of the experiment. Fluorescence intensity of manually outlined cell bodies and neurites was measured as a function of time. The intensity was further calculated by dividing the intensity at a given time point by the intensity at time point 0 (baseline level of time point 0 was set as 1). Statistical differences in reporter expression between groups were assessed by ANOVA in the StatView program (SAS Institute), followed by Fisher's LSD post hoc tests. The level of significance was set at $p \leq 0.05$.

Constructs. The CAMKII $\alpha$-5'-UTR-myr.EGFP$3^{\prime}$-UTR construct was a gift from Dr. E. Schuman (California Institute of Technology, Pasadena, CA) (Aakalu et al., 2001), and the sequence was verified using standard sequencing procedures. We first cloned Venus into the position of enhanced green fluorescent protein (EGFP). The Venus fragment was created using the following PCR primers: $5^{\prime}$-ctagtctagagtgagcaagggcgaggagc $-3^{\prime}$ (forward primer) and $5^{\prime}$ ttttccttttgcggccgcgaattcctacttgtacagctcgtc- $3^{\prime}$ (reverse primer), and Venus was then digested with $X b a \mathrm{I}$ and EcoRI and ligated into the CAMKII $\alpha-5^{\prime}$-UTR-myr.EGFP-3'-UTR construct with $X b a \mathrm{I}$ and NotI. To generate CAMKII $\alpha-5^{\prime}$-UTR-myr.Venus, the above construct was digested with NotI and ApaI to excise the $3^{\prime}$ UTR, and the vector was religated using Klenow fragment and blunt end ligation.

Virus production. AAV (serotype 8) was generated using plasmid triple-transfection of the AAV vector plasmid, packaging (encoding rep and cap genes), and adenovirus miniplasmid into HEK293A cells. Viral particles were purified by iodixanol gradient. Concentrated virus was isolated and titered by dot blot hybridization yielding $6.1 \times 10^{12}$ particles/ml (virus was provided by the Harvard Gene Therapy Initiative, Harvard Medical School, Boston, MA).

Animals and surgical procedures. APPswe/PS1dE9-transgenic mice at 9-11 months of age (APP/PS1; obtained from The Jackson Laboratory) and nontransgenic control mice were used in this study (eight transgenic and eight controls) (Jankowsky et al., 2004). APP/PS1 mice start to develop plaques in the neocortex between 5 and 7 months of age. All animal work conformed to National Institutes of Health and institutional guidelines. For intracortical injections of AAV containing the gene for Venus, mice were anesthetized with avertin (1.3\% 2,2,2-tribromethanol, $0.8 \%$ tert-pentylalcohol; $250 \mathrm{mg} / \mathrm{kg}$ ) and placed in a stereotaxic apparatus. The surgical site was sterilized with betadyne and isopropyl alcohol, and an incision was made in the scalp along the midline. Burr holes were drilled in the scull $1 \mathrm{~mm}$ lateral to bregma bilaterally. Using a Hamilton syringe, 
$3 \mu \mathrm{l}$ of virus was injected $1 \mathrm{~mm}$ deep at a rate of $0.2 \mu \mathrm{l} / \mathrm{min}$. After the injection, the scalp was sutured, and the mouse recovered from anesthesia on a heating pad.

After a 3 week incubation period to allow expression of Venus in neurons, one-half of the mice ( $n=4$ /group) were assigned to novel environment exploration without showing any signs of motor deficits and behavioral differences between transgenic and nontransgenic mice. These mice were transferred to a new cage containing three objects and allowed to explore the new environment for $30 \mathrm{~min}$ before they went back to their home cages for $20 \mathrm{~min}$ before they were euthanized with an overdose of avertin $(400 \mathrm{mg} / \mathrm{kg})$, transcardially perfused with ice-cold PBS and 4\% PFA, and the brains fixed in $4 \%$ paraformaldehyde in phosphate buffer with $15 \%$ glycerol cryoprotectant.

Immunohistochemistry. The brains were frozen and $30 \mu \mathrm{m}$ thick serial coronal sections were cut on a freezing sliding microtome. Sections were immunostained with the polyclonal antibody against GFP (ab 6556, 1:5000; Abcam), followed by secondary anti-rabbit conjugated to Alexa 488 (Molecular Probes). Images were acquired using a Zeiss LSM510 Meta laser scanning confocal microscope equipped with a $488 \mathrm{~nm}$ argon laser and 543 helium/neon laser and a 720-930 nm chameleon mode locked ti/saph laser. All images were acquired in $1 \mu \mathrm{m}$ sections, and $z$-series were set to span the entire neuronal volume. Image analysis was conducted on $z$-compressed image stacks. Contrast and intensity parameters were kept constant across all image acquisitions. By necessity, some soma and proximal dendrites had saturated image intensity to allow visualization of the entire dendritic tree. This saturation effect will underestimate any observed effects of genotype or enrichment. For cortical postmortem intensity measurements, 20 images of fields of Venus-infected neurons were taken per animal, and fluorescence intensity was measured using Adobe Photoshop 7 software (Adobe). Adobe Photoshop was used to convert the average GFP/ Venus fluorescence of each neuron to average pixel intensity. Data were expressed as mean $\pm \mathrm{SD}$ from the mean. Group means were compared using Student's $t$ test or ANOVA in the StatView program (SAS Institute). The level of significance was set at $p \leq 0.05$.

\section{Results}

Neuronal activity can be measured

\section{via dendritic protein synthesis in cultured neurons}

To measure neuronal activity in living neurons, we used a GFP reporter in which the coding sequence is flanked by the $5^{\prime}$ - and $3^{\prime}$-UTRs of CAMKII $\alpha$ (Aakalu et al., 2001). Previous work has shown that the $3^{\prime}$ - and $5^{\prime}$-UTR of the CAMKII $\alpha$ mRNA contains information sufficient for its dendritic localization (Mayford et al., 1996; Mori et al., 2000) and that BDNF stimulates protein synthesis especially in dendrites (Aakalu et al., 2001). In an initial experiment, we transfected primary neurons with the GFP reporter and measured fluorescent intensity over time after BDNF application to the media. In most untreated neurons, expression of the reporter was relatively weak in the cell body and neurites (Fig. 1a). However, neurons that were exposed to BDNF exhibited an increase in GFP synthesis that was evident in both the cell body and the neurites within tens of minutes, confirming previously published work (Aakalu et al., 2001) (Fig. 1b,c). The presence or absence of a myristoylation site did not alter these effects (data not shown). In an effort to enhance the signal for in vivo experiments in the mouse brain, we replaced GFP by Venus and sequentially decreased the size of the $3^{\prime}$-UTR from 3100 to 420 bp. Venus is a variant of yellow fluorescent protein (YFP) and the brightest yellow variant to date (Nagai et al., 2002). A small (420 bp) 3'-UTR containing construct still responded to BDNF treatment (Fig. $1 g, h$ ) and was small enough to be efficiently packaged into AAV. As expected, untreated control neurons remained stable (Fig. 1d,e), but BDNF-treated neurons had significantly higher intensity in cell bodies and neurites already evident after 30 min of BDNF exposure (Fig. $1 f, i$ ).

To further characterize and determine the necessity of the $3^{\prime}$-UTR in directing protein synthesis to the dendrites in an activity-dependent manner, we generated another construct completely missing the $3^{\prime}$-UTR. In contrast to the abovementioned effect of BDNF on transfected neurons, those neurons were unresponsive and did not differ from untreated control neurons (supplemental Fig. 1, available at www.jneurosci.org as supplemental material) showing that the $3^{\prime}$-UTR of CAMKII $\alpha$ is a critical element in activity-dependent neuronal protein synthesis.

\section{Neurons from APP/PS1 mice have decreased neuronal} activity and are unaffected by environmental stimulation We next generated a $3^{\prime}$-UTR CAMKII $\alpha$ Venus $5^{\prime}$-UTR AAV and performed intracortical injections into the somatosensory cortex of adult APP/PS1 and wild-type mice. APP/PS1 mice, a transgenic model of $\mathrm{AD}$, overexpress mutant human APP and PS1, leading to the robust deposition of amyloid- $\beta$ plaques. After an 

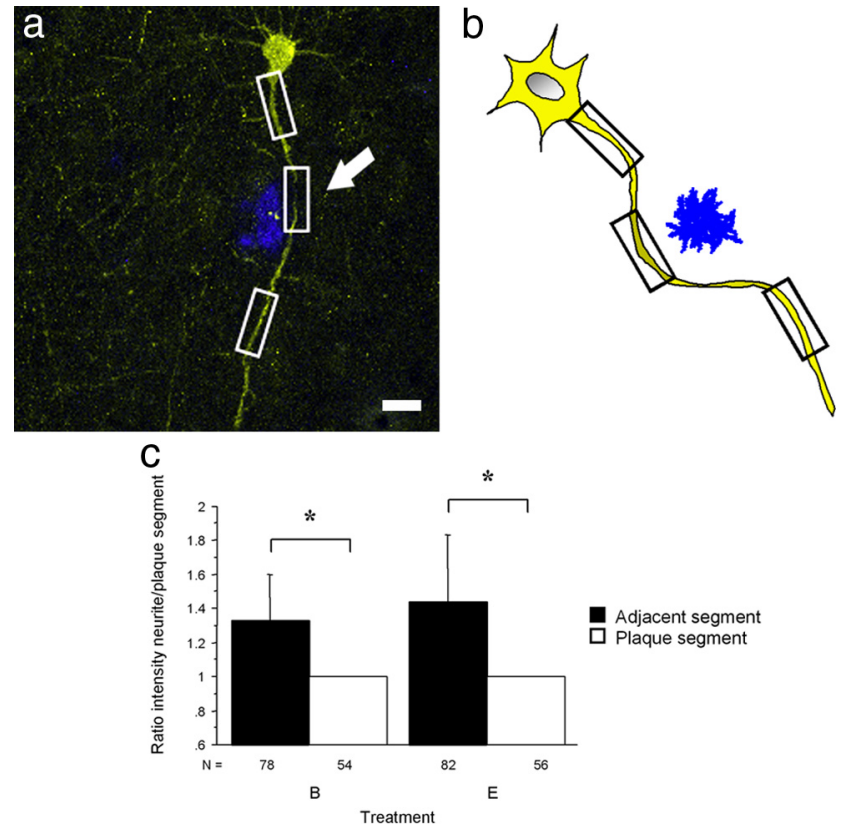

Figure 3. Individual dendritic segments in close proximity to a plaque have decreased neuronal activity. $\boldsymbol{a}, \boldsymbol{b}$, A neuron and a schematic illustration of a neuron filled with Venus in the vicinity of a plaque (shown in blue). White and black boxes mark the segments where intensity was measured. Note the decreased intensity in the segment close to the plaque indicated by the arrow. c, Dendritic segments farther from the plaque had a statistically significant higher intensity when compared with the dendritic segments nearest to the plaque in unstimulated as well as in stimulated neurons ( $* p<0.0001)$. Scale bar: $\boldsymbol{a}, 10 \mu \mathrm{m}$.

incubation time of 3 weeks that allowed viral transduction of neurons, one-half of the mice explored a new enriched environment for 30 min before the brains were analyzed postmortem by immunostaining to label Venus-expressing neurons. Similar injection of 3 '-UTR CAMKII $\alpha$ Venus 5' -UTR AAV under identical conditions surprisingly led to decreased neuronal Venus fluorescence intensity in the vicinity of plaques when compared with wild-type neurons (Fig. 2a,e). Moreover, neuronal cell bodies distant $(>100 \mu \mathrm{m})$ from plaques were clearly brighter $(p<$ 0.0001 ) (Fig. $2 c, g, h$ ). This effect was also observed, perhaps even more dramatically, in individual dendritic segments near a plaque. Dendritic segments closest to a plaque were substantially dimmer than adjacent segments on either side $(p<0.0001)$ (Fig. $3 a-c)$. On the basis of this finding, we also tested whether the reporter would show a response if the transgenic mice were allowed to explore an enriched environment. In contrast to wildtype mice, neurons from APP/PS1-transgenic mice near plaques remained unaltered after environmental enrichment (Fig. $2 b, g, h)$, whereas cell bodies distant from a plaque $(>100 \mu \mathrm{m}$ away) have a detectable response to environmental stimulation (Fig. $2 d, g$ ). Thus, our data indicate that neurons in the vicinity of plaques have diminished neuronal function and are unresponsive to environmental stimulation, in contrast to neurons from the same mouse that are distant from a plaque, or, most obviously, from nontransgenic mice.

\section{Discussion}

In the current study, we re-engineered a reporter molecule previously shown to respond to BDNF application in primary neuronal culture to initially test the hypothesis that dendritic mRNA transport and local translation occur after behaviorally relevant stimuli in vivo. The $3^{\prime}$ - and 5'-UTR of CAMKII $\alpha$ encodes sequences that direct translocation to the dendrite and direct local translation (Aakalu et al., 2001; Sutton et al., 2004; Smith et al., 2005). In initial experiments, we found that the proximal $420 \mathrm{bp}$ of the 3 '-UTR contains sequences that are critical for activitydependent protein synthesis.

$\mathrm{AD}$ is a neurodegenerative disorder that is characterized by severe memory impairment due, in part, to synaptic dysfunction. Amyloid plaques are deposited throughout the cortex, but the role of plaques in neurodegeneration and in neural system function is uncertain and has recently been challenged by the idea that soluble forms of $\mathrm{A} \beta$, rather than plaques, are the toxic species since soluble oligomers are sufficient to induce deficits in LTP and behavior in rodent models (Shankar et al., 2008). In contrast, plaques are associated with inflammatory responses, neuronal morphological alterations, and disturbances in local calcium homeostasis (D'Amore et al., 2003; Spires et al., 2005; Busche et al., 2008; Kuchibhotla et al., 2008; Meyer-Luehmann et al., 2008). We used the CAMKII $\alpha$ UTR Venus AAV to test the hypothesis that plaques would be associated with dysfunction of neural system integration after behaviorally relevant stimuli at the level of the dendritic segment and cell body.

Surprisingly, we found only very limited neuronal Venus expression in plaque-bearing APP/PS1-transgenic mice in contrast to the robust fluorescence in wild-type neurons. Neurons and dendrites distant from a plaque in the APP/PS1 cortex showed higher levels of the Venus reporter, arguing for a plaque-specific effect on the microenvironment near an amyloid deposit. Our data argue that reduced reporter synthesis occurs in transgenic neurons as a result of diminished activation near plaques, rather than as a result of technical issues of reporter expression in transgenic animals, since 1) analysis showed that the least fluorescence intensity in transgenic dendrites occurs in the vicinity of plaques so that the portion of the dendrite closest to the plaque has less Venus than the average of the segments proximal and distal to the plaque of the same dendrite; 2) neurons whose cell bodies are close to plaques have diminished reporter expression compared with neurons in the same mice only a $100 \mu \mathrm{m}$ away but distant from a plaque; and 3) a differential response to environmental enrichment was observed in neurons close to or far from plaques.

APP-transgenic mice show reduced expression of Arc and other immediate early genes known to be activity dependent (Dickey et al., 2004; Palop et al., 2005). We observed that environmental enrichment induced a stimulation of local dendritic reporter synthesis in the somatosensory cortex in wild-type mice. Although transgenic mice likewise explored the enriched environment, neurons and isolated dendritic segments near plaques failed to respond upon behavioral stimulation. The deficit in activation specifically in dendritic segments likely leads to impaired integration of somatosensory signaling, impaired reporter activity in the cell bodies, and failure of broader neural system function as the impairment in dendritic integration plays out throughout the arborizations associated with these dendritic elements.

In summary, our data show that 1) mRNA translocates into dendrites and can be translated there in response to physiologic stimuli in vivo; 2) this property can be used to monitor neural activity within individual dendritic segments, opening a new level of structure-function relationships to understand signal integration properties on a subcellular basis; and 3 ) this tool also allowed us to explore the consequences of $\mathrm{A} \beta$ deposits on neural system function in a mouse model of AD. Amyloid plaques appear to be a focal lesion, around which there is impaired response, suggesting that plaques [and potentially elevated $\mathrm{A} \beta$-soluble species in equilibrium near plaques (Koffie et al., 2009)] directly impact 
neurons' responses to physiological stimuli. Together with recent observations showing alteration of neuronal calcium homeostasis near plaques, the presence of morphological axonal and dendritic alterations and dendritic spine loss specifically near plaques (D'Amore et al., 2003; Spires et al., 2005; Kuchibhotla et al., 2008; Meyer-Luehmann et al., 2008), and electrophysiological data implicating plaque-dependent disruption of neural system integration (Stern et al., 2004), we conclude that individual plaques serve as focal pathophysiological lesions with a wide-ranging impact on nearby and distant neural systems. We postulate that numerous plaques thus impair neural system function by altering dendritic integration of information in a distributed manner throughout the cortex, preventing the activation of otherwise intact neuronal structures. This construct may be the underlying basis of changes in neural system activation observed by functional magnetic resonance imaging (fMRI) studies of $A D$ patients and help explain the concordance between the pattern of altered activation in resting fMRI studies of $\mathrm{AD}$ patients and amyloid plaque deposition (Buckner et al., 2005; Buckner et al., 2009).

\section{References}

Aakalu G, Smith WB, Nguyen N, Jiang C, Schuman EM (2001) Dynamic visualization of local protein synthesis in hippocampal neurons. Neuron 30:489-502.

Buckner RL, Snyder AZ, Shannon BJ, LaRossa G, Sachs R, Fotenos AF, Sheline YI, Klunk WE, Mathis CA, Morris JC, Mintun MA (2005) Molecular, structural, and functional characterization of Alzheimer's disease: evidence for a relationship between default activity, amyloid, and memory. J Neurosci 25:7709-7717.

Buckner RL, Sepulcre J, Talukdar T, Krienen FM, Liu H, Hedden T, AndrewsHanna JR, Sperling RA, Johnson KA (2009) Cortical hubs revealed by intrinsic functional connectivity: mapping, assessment of stability, and relation to Alzheimer's disease. J Neurosci 29:1860-1873.

Busche MA, Eichhoff G, Adelsberger H, Abramowski D, Wiederhold KH, Haass C, Staufenbiel M, Konnerth A, Garaschuk O (2008) Clusters of hyperactive neurons near amyloid plaques in a mouse model of Alzheimer's disease. Science 321:1686-1689.

Cotman CW, Geddes JW, Kahle JS (1990) Axon sprouting in the rodent and Alzheimer's disease brain: a reactivation of developmental mechanisms? Prog Brain Res 83:427-434.

D’Amore JD, Kajdasz ST, McLellan ME, Bacskai BJ, Stern EA, Hyman BT (2003) In vivo multiphoton imaging of a transgenic mouse model of Alzheimer disease reveals marked thioflavine-S-associated alterations in neurite trajectories. J Neuropathol Exp Neurol 62:137-145.

Dickey CA, Gordon MN, Mason JE, Wilson NJ, Diamond DM, Guzowski JF, Morgan D (2004) Amyloid suppresses induction of genes critical for memory consolidation in APP + PS1 transgenic mice. J Neurochem 88:434-442.

Jankowsky JL, Slunt HH, Gonzales V, Jenkins NA, Copeland NG, Borchelt DR (2004) APP processing and amyloid deposition in mice haploinsufficient for presenilin 1. Neurobiol Aging 25:885-892.

Knowles RB, Wyart C, Buldyrev SV, Cruz L, Urbanc B, Hasselmo ME, Stanley HE, Hyman BT (1999) Plaque-induced neurite abnormalities: implica- tions for disruption of neural networks in Alzheimer's disease. Proc Natl Acad Sci U S A 96:5274-5279.

Koffie RM, Meyer-Luehmann M, Hashimoto T, Adams KW, Mielke ML, Garcia-Alloza M, Micheva KD, Smith SJ, Kim ML, Lee VM, Hyman BT, Spires-Jones TL (2009) Oligomeric amyloid $\beta$ associates with postsynaptic densities and correlates with excitatory synapse loss near senile plaques. Proc Natl Acad Sci U S A 106:4012-4017.

Kuchibhotla KV, Goldman ST, Lattarulo CR, Wu HY, Hyman BT, Bacskai BJ (2008) $\mathrm{A} \beta$ plaques lead to aberrant regulation of calcium homeostasis in vivo resulting in structural and functional disruption of neuronal networks. Neuron 59:214-225.

Le R, Cruz L, Urbanc B, Knowles RB, Hsiao-Ashe K, Duff K, Irizarry MC, Stanley HE, Hyman BT (2001) Plaque-induced abnormalities in neurite geometry in transgenic models of Alzheimer disease: implications for neural system disruption. J Neuropathol Exp Neurol 60:753-758.

Mayford M, Baranes D, Podsypanina K, Kandel ER (1996) The 3'untranslated region of CaMKII $\alpha$ is a cis-acting signal for the localization and translation of mRNA in dendrites. Proc Natl Acad Sci USA 93:13250-13255.

Meyer-Luehmann M, Spires-Jones TL, Prada C, Garcia-Alloza M, de Calignon A, Rozkalne A, Koenigsknecht-Talboo J, Holtzman DM, Bacskai BJ, Hyman BT (2008) Rapid appearance and local toxicity of amyloid- $\beta$ plaques in a mouse model of Alzheimer's disease. Nature 451:720-724.

Mori Y, Imaizumi K, Katayama T, Yoneda T, Tohyama M (2000) Two cisacting elements in the $3^{\prime}$ untranslated region of $\alpha$-CaMKII regulate its dendritic targeting. Nat Neurosci 3:1079-1084.

Nagai T, Ibata K, Park ES, Kubota M, Mikoshiba K, Miyawaki A (2002) A variant of yellow fluorescent protein with fast and efficient maturation for cell-biological applications. Nat Biotechnol 20:87-90.

Palop JJ, Chin J, Bien-Ly N, Massaro C, Yeung BZ, Yu GQ, Mucke L (2005) Vulnerability of dentate granule cells to disruption of arc expression in human amyloid precursor protein transgenic mice. J Neurosci 25:9686-9693.

Phinney AL, Deller T, Stalder M, Calhoun ME, Frotscher M, Sommer B, Staufenbiel M, Jucker M (1999) Cerebral amyloid induces aberrant axonal sprouting and ectopic terminal formation in amyloid precursor protein transgenic mice. J Neurosci 19:8552-8559.

Shankar GM, Li S, Mehta TH, Garcia-Munoz A, Shepardson NE, Smith I, Brett FM, Farrell MA, Rowan MJ, Lemere CA, Regan CM, Walsh DM, Sabatini BL, Selkoe DJ (2008) Amyloid- $\beta$ protein dimers isolated directly from Alzheimer's brains impair synaptic plasticity and memory. Nat Med 14:837-842.

Smith WB, Starck SR, Roberts RW, Schuman EM (2005) Dopaminergic stimulation of local protein synthesis enhances surface expression of GluR1 and synaptic transmission in hippocampal neurons. Neuron 45:765-779.

Spires TL, Meyer-Luehmann M, Stern EA, McLean PJ, Skoch J, Nguyen PT, Bacskai BJ, Hyman BT (2005) Dendritic spine abnormalities in amyloid precursor protein transgenic mice demonstrated by gene transfer and intravital multiphoton microscopy. J Neurosci 25:7278-7287.

Stern EA, Bacskai BJ, Hickey GA, Attenello FJ, Lombardo JA, Hyman BT (2004) Cortical synaptic integration in vivo is disrupted by amyloid- $\beta$ plaques. J Neurosci 24:4535-4540.

Sutton MA, Wall NR, Aakalu GN, Schuman EM (2004) Regulation of dendritic protein synthesis by miniature synaptic events. Science 304:19791983. 